\title{
Measurement of displacement in the micrometer range using speckle pattern correlation in multimode fibers
}

\author{
M. Lomer ${ }^{1}$, J. Abascal ${ }^{1}$, J. Castrellon-Uribe ${ }^{2}$, J.M. Lopez-Higuera ${ }^{1}$ \\ ${ }^{1}$ Photonics Engineering Group, University of Cantabria, Santander 39005, Spain \\ ${ }^{2}$ CIICAP, Universidad Autónoma del Estado de Morelos, C.P. 62209 Cuernavaca Morelos Mexico.
}

\begin{abstract}
The displacement measurement in the micrometer range using the speckle pattern correlation in optical fibers is described. The high sensitivity of a multimode fiber when propagating a coherent light causes any variation in the distribution of the speckle pattern is proportional to the disturbance which it originates. The speckle pattern is caused by interference between modes and the shift mechanism is due to coupling between modes and micro-induced redistribution curvature applied to the fiber externally. The movement is produced by a transducer containing microbends pressure induced on the fiber. Experimental results are presented using three types of multimode plastic optical fiber. In the signal generated from the change in the speckle pattern observed with relatively good reproducibility, so that sensor systems could be used.
\end{abstract}

Keywords: Displacement measurement, multimode fiber, speckle pattern, plastic optical fiber

\section{INTRODUCTION}

The multimode optical fibers having a core diameter much larger than single mode fibers and are therefore much easier to use in telecommunications and in sensor technology. Particularly, plastic optical fibers (POF) have core diameters multimodal ranging from $50 \mathrm{~m}$ to $1000 \mu \mu \mathrm{m}$ have excellent flexibility and adaptability, easy to manipulate, robust and good mechanical strength. Multimode fibers bear large amounts of modes, which propagate with different phase velocities, and if the light is coherent in the output end of the fiber can be observed on a screen a highly structured intensity distribution, called speckle pattern [1], pellets composed of bright and dark light. This phenomenon is attributed to interference between modes [1-2]. The amount of speckle in the pattern is approximately equal to the number of modes supported by the fiber. The speckle pattern in fiber is extremely sensitive to environmental perturbations. That is, the bright light granules slowly varying effect of ambient temperature variations, but the total current is constant. Processing the images capture the speckle patterns in time allow to extract information on the cause of the disturbance. Based on this phenomenon, vibration sensors have been reported [2], displacement [3-4], distance [5], is breaking concrete structures [6] and blood flow [7]. Particularly displacement sensors using correlation technique speckle pattern are potentially interesting in medicine and biology or industry which require high precision tools. The work reported by Garcia-Valenzuela $\mathrm{Yu}$ and demonstrated the ability to measure displacement in the micrometer range using the correlation speckle patterns with offset [3.4]. The movement is induced by a micro-device by transverse displacement curves on a multimode fiber numerical aperture of 0.2 and $50 \mathrm{~cm}$ length [3] and a disruptive force in the center of the glass fiber $125 \mu \mathrm{m}$ core diameter within an aluminum structure [4]. These works showed a sensitivity of $0.1 \mu \mathrm{m}$ over a measured range of $20 \mu \mathrm{m}$ and $30 \mathrm{~nm}$ over a range of $500 \mathrm{~nm}$, respectively.

In this paper presents the measurement of displacement using the correlation between changes in distribution of the speckle patterns and disturbance in multimode fiber. A device with controlled micro-bends and is used to induce micrometric displacement, and therefore changes in the speckle pattern at the fiber output. Speckle patterns are recorded by a CCD camera and processed to extract the relevant information to the displacement. Experimental measurements were performed in plastic optical fibers with core diameters of $50 \mu \mathrm{m}, 240 \mu \mathrm{m}$ and $980 \mu \mathrm{m}$. Shows the influence of the number of modes propagated in the fiber on the sensitivity and measuring range. Experimental results are presented and discussed.

*lomerm@unican.es; phone 34 942201495; fax 34 942201873; web: unican.es

Fifth European Workshop on Optical Fibre Sensors, edited by Leszek R. Jaroszewicz, Proc. of SPIE Vol. 8794, $879445 \cdot$ • (2013 SPIE · CCC code: 0277-786X/13/\$18 - doi: 10.1117/12.2026804 


\section{SPECKLE PATTERNS IN MULTIMODE FIBER}

Multimode fibers can spread a large number of modes with different phase velocities. The propagation modes corresponding to different optical paths used by the beams coupled into the fiber suffer different phase delays. The output field distribution consists of a sum of all individual contributions of each mode. If the contributions phase delay varies over $2 \pi$ radians, having a sufficiently coherent source then, the interference effects are well structured and can be observed in the intensity distribution through the end of the fiber. The number of modes, M, which supports an optical fiber break index is given by the expression [8], $M=V^{2} / 2$, where $V$ is called normalized frequency given by, $V=2 \pi a\left(n_{c o}^{2}-n_{c l}^{2}\right) / \lambda$, where $a$ is the core radius, $\lambda$ wavelength the laser and $n_{c o}$ and $n_{c l}$ are the refractive indexes of core and shell, respectively. For a gradient index fiber, the number of modes is: $M \approx V^{2} / 4$. For example, if all modes are excited in the multimode fiber step-index, $n_{c o}=1.492$ and $n_{c l}=1.402$, and core radius $a=125$ and $490 \mu m$, the number of modes that would be about 200,000 and 3 million modes, respectively, when a He-Ne laser $(\lambda=0.6328 \mu m)$ is used. Due to the number of speckle dots present in a pattern is approximately equal to $M$, a speckle pattern adaptable to the needs of the measured variable can be obtained just by choosing a fiber with a suitable diameter and consequently by determining the size of the speckle dots.

\section{EXPERIMENTAL SETUP AND PROCEDURE}

Figure 1 shows the configuration of the experimental system for measuring displacement. The characteristics of the POF used are described in Table 1. In all cases we used a fiber length of $2 \mathrm{~m}$. The step-index POFs, with core diameter of 240 $\mathrm{mm}$ and $980 \mathrm{~mm}$ manufactured by Toray ${ }^{\circledR}$ are of the same material and have the same numerical aperture. The gradedindex POF core material is a polymer perfluorinated (PF) manufactured by Chromis Fiberoptics ${ }^{\circledR}$, the maximum refractive index in the fiber axis, is 1.35 and the index of the cladding is 1.34. Experimental measurements were made with a HeNe laser source $(\lambda=0.6328 \mu \mathrm{m})$. Light is directly coupled to the fiber and the output end is connected to a CCD camera that records the speckle patterns during displacement. A transducer device is incorporated for micrometric displacement. The resulting transverse displacement in the longitudinal elongation of the fibers which affects the phase of the fiber modes and modes couplings occur randomly. The CCD camera located at the output extreme capture images of the speckle patterns during displacement. The CCD camera sends images to a computer that is responsible for processing the speckle patterns. Fig. 2 shows a photograph of the speckle patterns obtained in the output end of the three POF described in Table 1. It can be observed visually each speckle area depends on the fiber diameters. One can anticipate that to effect sensors utilize this phenomenon depends on the nature of the disturbance to realize on the fiber. The camera captures 30 images per second, so each displacement of 1 second is recorded in a video image. The data are processed by the method of the co-occurrence matrix [9]. The co-occurrence matrix describes the frequency with which a gray level is displayed in a spatial neighborhood relationship with another gray level in an image. This matrix summarizes how often think of two neighboring pixels separated by a distance, given by displacement. The dimension of the matrix is the number of quantization levels of gray of the image. Therefore, by this method we can relate the displacement $\mathrm{d}$ in the speckle patterns made with the mechanical displacement transducer.

Table 1. Plastic optical fibers used in the measurement of displacement

\begin{tabular}{|l|c|c|c|c|c|}
\hline Fiber core material & $\begin{array}{c}\text { Core/cladding } \\
\text { diameter }(\mu \mathrm{m})\end{array}$ & $\begin{array}{c}\text { Refractive } \\
\text { index }\left(\mathrm{n}_{\mathrm{co}}\right)\end{array}$ & $\begin{array}{c}\text { Numerical } \\
\text { Aperture }\end{array}$ & Weight $(\mathrm{g} / \mathrm{m})$ & Number modes* \\
\hline PMMA (step-index) & $980 / 1000$ & 1.49 & 0.5 & 1.0 & 3 '082,691 \\
\hline PMMA (step-index) & $240 / 250$ & 1.49 & 0.5 & 0.01 & 184,884 \\
\hline $\begin{array}{l}\text { Perfluorinated } \\
\text { graded-index) }\end{array}$ & $50 / 500$ & $(1.34-1.35)$ & 0.2 & ---- & 665 \\
\hline
\end{tabular}

*calculated with $\lambda=0.6328 \mu \mathrm{m}$. 


\section{RESULTS AND DISCUSSION}

To determine the co-occurrence matrix and made displacement relate to changes in the speckle pattern is used the "graycomatrix" incorporating the Matlab program. We then use the "graycoprops" allows find four basic descriptors defined by Harilick [9]. When you call the function in the co-occurrence matrix, it returns descriptors: contrast, homogeneous, correlation and energy. Of these descriptors, the best answer is provided by the energy. Here, we use the term "energy" only as a mathematical descriptor. As we proceed to perform a deeper analysis of the property and effectively characterize the measurements and their relation to induced displacement in the fiber. In Figure 3 are plotted the results obtained with the fibers of different core diameters. The curves have been adjusted by polynomials to a linear variation. This analysis takes into account the linear part of the variation. The ends of the curves have been filtered can therefore not be considered in a possible practical implementation. Comparing the slopes of the three fibers, the result is observed in Fig. 3(a) for the fiber of $980 \mu \mathrm{m}$ core diameter has the best relationship energy/displacement $(0.011 / \mu \mathrm{m})$ measurement over a range of displacement approximately $100 \mu \mathrm{m}$.

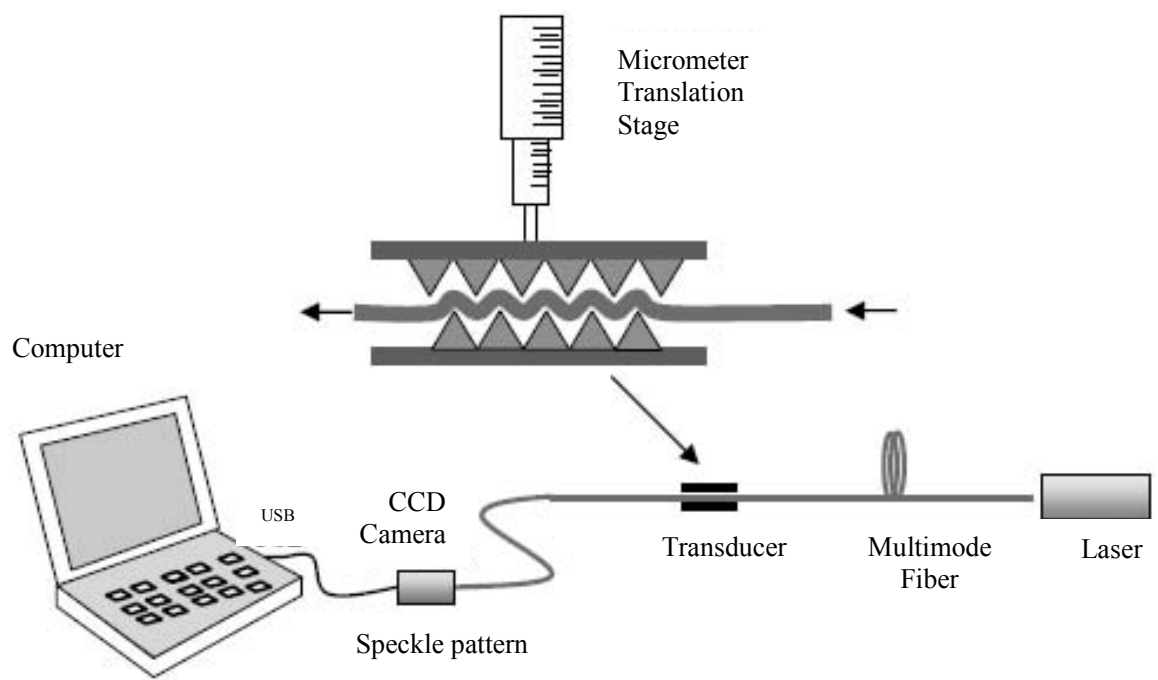

Figure 1. Experimental setup for the measurement of displacement by correlation speckle patterns and the disturbance induced micro-curvatures of the fiber.
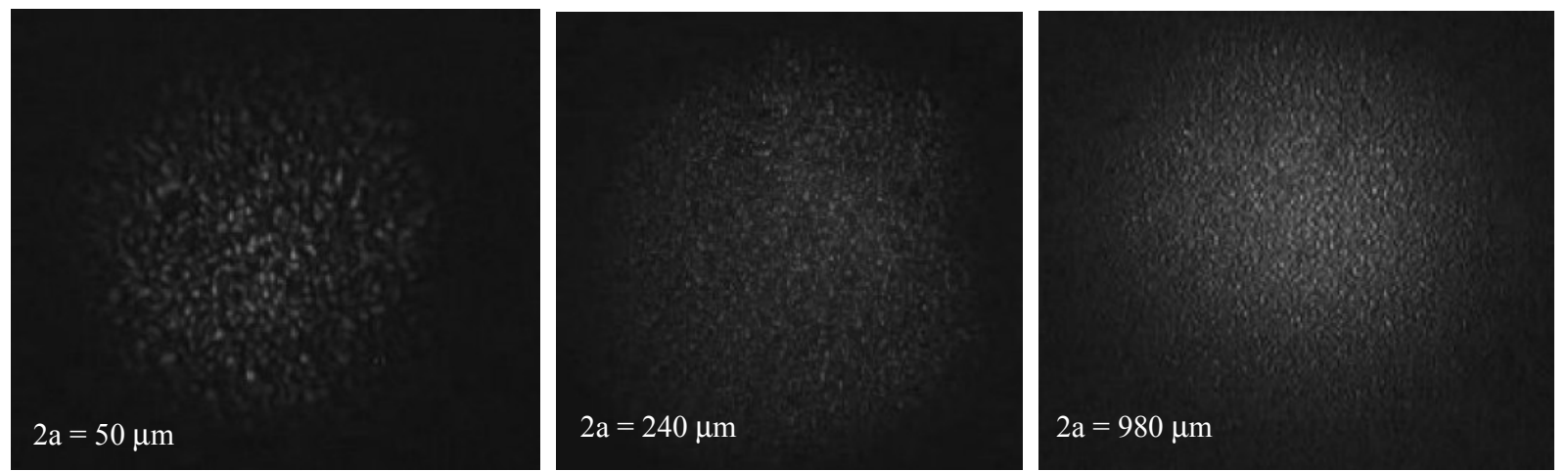

Figure 2. Photography speckle patterns recorded in output POF fibers with core diameters of $50 \mu \mathrm{m}, 240 \mu \mathrm{m}, 980 \mu \mathrm{m}$. 


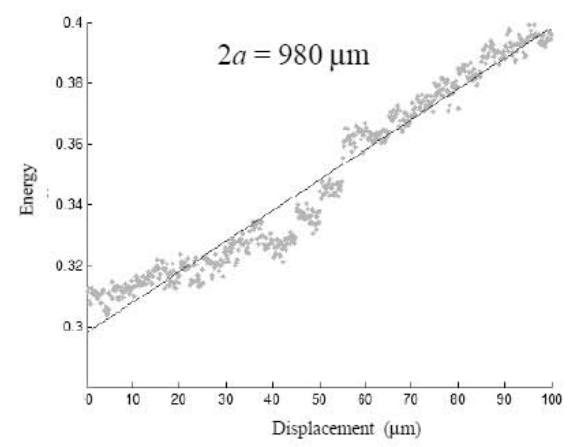

(a)

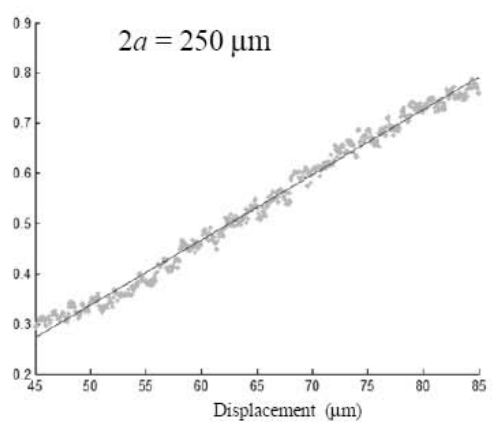

(b)

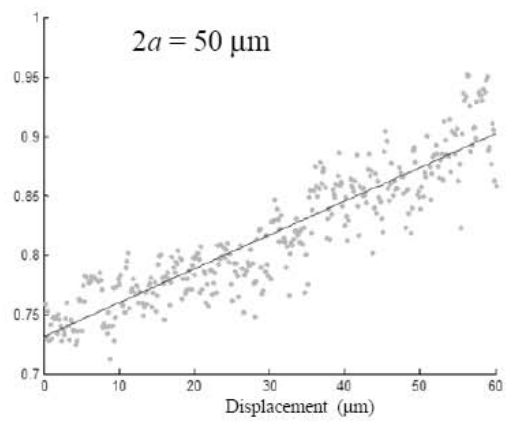

(c)

Fig. 3. Energy co-occurrence matrix as a function of displacement obtained from the speckle patterns into 3 types of multimode fibers.

The curve of Figure 3(b) has a slope slightly lower $(0.0117 / \mu \mathrm{m})$ within a range of displacement of $40 \mathrm{~mm}$. But the dispersion of the points of correlation calculated is virtually straight according to the adjustment. The curve of Fig. 3(c) has a slope over a range of $0.003 / \mu \mathrm{m}$ measured over a measuring range of $60 \mathrm{~mm}$. The dispersion of the measurement points are quite large, therefore induce a greater error.

\section{CONCLUSION}

We measured the displacement in micrometer range using the correlation speckle patterns at different multimode fiber core diameters. The best results were obtained with $240 \mu \mathrm{m}$ fiber diameter in the range of displacement of $40 \mu \mathrm{m}$ with good accuracy. The fiber diameter of $980 \mu \mathrm{m}$ is more robust and can measure a range of displacement up to $100 \mu \mathrm{m}$ with moderate accuracy. Experimental measurements are repetitive and could be implemented in systems of low cost sensors.

The authors acknowledge the Spanish government for supporting this work through the TEC2010-20224-CO2-02 projects and with the grant AP2009-1403.

\section{REFERENCES}

[1] J.W. Goodman, Speckle Phenomena in Optics, Roberts and Company, Chapter 7, Englewood, Colorado, (2007).

[2] W.B. Spillman,.Jr., B.R. Kline, L.B. Maurice, and P.L. Fuhr, "Statistical-mode sensor for fiber optic vibration sensing uses", Applied Optics, 28, 3166-3176, (1989).

[3] F.T.S. Yu, M. Wen, S. Jing, and C.M. Uang, "Submicrometer displacement sensing using inner-product multimode fiber speckle fields", Applied Optics, 32, 4685-4689, (1993).

[4] A. Garcia-Valenzuela and M. Tabib-Azar, "Fiber-Force and displacement sensor based on speckle detection with $0.1 \mathrm{nN}$ and $0.1 \AA ”$ ", Sensor and Actuators A, 36, 199-208, (1993).

[5] Y. Kitagawa, and A. Hayashi, "Fiber-optic sensor for distance and velocity measurements using speckle dynamics", Applied Optics, 24, 955-959, (1985).

[6] Z. Zhang, and F. Ansari, "Fiber-optic laser speckle-intensity crack sensor for embedment in concrete", Sensors and Actuators A-126, 107-111, (2006).

[7] G. Dietsche, M. Ninck, C. Ortolf, J. Li, F. Jaillon, and T. Gisler, "Fiber-based multispeckle detection for timeresolved diffusing-wave spectroscopy: characterization and application to blood flow detection in deep tissue", Applied Optics, 46, 8506-8514, (2007).

[8] G. Kaiser, Optical Fiber Communications, McGraw-Hill, Second Edition, Chapter 2, NY (1991).

[9] R.M. Haralick, K. Shanmugan and I. Dinstein, "Textural features for image classification, IEEE Transaction on System, Man and Cybernetics, Vol. SMC-3(6), 610-621 (1973). 